\title{
Serum Adiponectin Level Association with Breast Cancer Risk: Evidence from a Case-Control Study
}

\author{
Syed Danish Haseen Ahmed ${ }^{1 *}$, Aziza Khanam², Naheed Sultan ${ }^{3}$, Farah Idrees ${ }^{3}$, \\ Naheed Akhter ${ }^{2}$
}

\begin{abstract}
Background: Adiponectin is a protein, synthesized by adipose tissue, which has a questionable role in breast cancer pathogenesis. This study focused on serum levels of adiponectin among cases and controls, and its possible association with breast cancer risk. Materials and Methods: This case-control study covered 175 diagnosed breast cancer patients with positive histopathology from Breast Clinic, surgical unit-1, Civil Hospital, Karachi and 175 healthy controls from various screening programs. Blood samples were obtained after an overnight fast and later analyzed. Results: Fasting blood glucose was significantly raised, while serum adiponectin was significantly decreased in breast cancer cases when compared to control subjects. To find the precise influence of serum adiponectin on the risk of breast cancer, subjects were divided in to quartiles based on serum levels of adiponectin. It is observed that risk of breast cancer decreased with rising quartile. Q2 (25th-50th) had the highest $\mathrm{OR}=1.76$ (CI: 0.93- 3.34), $\mathrm{Q3}$ (50th-75th) has $\mathrm{OR}=0.89$ (CI: 0.48-1.64), while subjects in the highest quartile Q4 ( $>75$ th) had significantly $(p<0.05)$ decreased risk of breast cancer having $\mathrm{OR}=0.06$ (CI: 0.02-0.15), when compared to the lowest quartile. Conclusions: We demonstrated a statistically significant association of elevated serum adiponectin with decreased risk of breast cancer. This signifies a protective role of adiponectin via an intricate mechanism of masking mitogenic growth factors in breast carcinogenesis.
\end{abstract}

Keywords: Adiponectin - breast cancer - risk

Asian Pac J Cancer Prev, 16 (12), 4945-4948

\section{Introduction}

Breast cancer in women is a major health problem worldwide. It is the most common cancer diagnosed and cause of death due to cancer among women (Torre et al., 2015). According to the recent estimates for year 2012 by International Agency for Research on Cancer (IARC), 1.67 million incident cases and 521,900 deaths were due to breast cancer globally (Ferlay et al., 2015). Not only western countries (Leclere et al., 2013) but also Asian population has also shown rise in breast cancer incidence (Keramatinia, 2014).

Adiponectin is a 244 amino acid protein synthesized by adipose tissue. Present exclusively in adipose tissue and blood, and interacts with its two receptors namely AdipoR1 and AdipoR2 (Yamauchi, 2003; Kadowaki and Yamauchi 2005). AdipoR1 is expressed mainly in skeletal muscles while AdipoR2 is expressed mainly in the liver. Both AdipoR1 and AdipoR2 contain G-protein coupled receptors with seven transmembrane domains (Yamauchi, 2003; Kadowaki et al., 2006).

Adiponectin has been investigated for its protective role against cancer. Adiponectin directly protects through binding selectively to several mitogenic growth factors, such as platelet-derived growth factor BB (PDGF-BB), basic fibroblast growth factor (FGF), and heparin binding epidermal growth factor-like growth factor (HB EGF) that can induce proliferation in many types of cells. This prevents the binding of mitogenic growth factors to the membrane receptors and thus stop cascade at a prereceptor level (Wang et al., 2005). Moreover, adiponectin may inhibit activation of nuclear factor- $x \mathrm{~B}(\mathrm{NF}-x \mathrm{~B})$, a transcription factor that up regulates vascular endothelial growth factor (VEGF) in breast cancer cells (Kelesidis et al., 2006).

Low serum adiponectin has been associated with high rate of obesity related cancer diseases, including breast cancers. The expression of AdipoR1 and R2 in breast cancer cells (Korner et al., 2007) has stipulated the role of serum adiponectin in breast cancer. The objective of this study is to test the hypothesis that elevated serum adiponectin level is associated with decreased risk of breast cancer. To keep this in mind odds ratio was determined to find the risk of breast cancer among quartiles based on serum adiponectin levels, while age, BMI, waist circumference and biochemical parameters were also compared between cases and control to observe the possible differences simultaneously.

${ }^{1}$ Biochemistry, medicine, dow medical college, DOW university of health sciences, ${ }^{2}$ Department of Biochemistry, University of Karachi, ${ }^{3}$ Department of Surgery, Dow Medical College and Civil Hospital Karachi, Dow University of Health Sciences, Karachi, Pakistan*For correspondence: danish-haseen@duhs.edu.pk 


\section{Materials and Methods}

This study was designed as a case-control study to identify the influence of Serum Adiponectin to the risk of breast cancer. It was conducted at Department of Biochemistry, University of Karachi from June 2010 to August 2014. Sample size was calculated by using frequency (34.6\%) of breast cancer in Pakistan indicated in a previous study (Bhurgri et al., 2004). Ethical approval was acquired from the ethics and research committee of the Department of Biochemistry, University of Karachi. Data of 175 diagnosed breast cancer patients with positive histopathology from Breast Clinic, Civil Hospital, Karachi and 175 controls from various screening programs were collected after their informed consent. Subjects undergone breast surgery, receiving chemotherapy or radiotherapy, on anti-estrogen drugs, hormones replacement therapy, contraceptive pills and subjects currently on insulin or anti diabetic treatment were excluded from the study.

Blood samples were obtained after an overnight fast by vein puncture under all aseptic measures. For premenopausal women blood samples were collected in the first week (early) of follicular phase of menstrual cycle to lower inconsistency among subjects. Serum was separated after clotting, centrifuged and stored at $-30^{\circ} \mathrm{C}$. Blood glucose was analyzed immediately using glucose oxidase kit provided by Merck, Germany, while serum adiponectin was analyzed later by ELISA kit manufactured by DIASource, Belgium.

Later data was entered and analyzed by using IBM SPSS 20 (IBM, 2011) statistical software and OpenEpi, Version 3, open source calculator (Dean, 2014). Mean values of age, BMI, waist circumference and biochemical parameters were compared by using student t-test. Where as odds ratio was estimated for finding out the risk of serum adiponectin for breast cancer.

\section{Results}

A total of 350 subjects were selected. All the subjects were distributed into two groups, 175 cases suffering from breast cancer and their age, sex and BMI matched 175 controls healthy subjects. There were $39.4 \%$ premenopausal subjects among cases while $47.4 \%$ in control group. Similarly $60.5 \%$ postmenopausal were among cases while $52.5 \%$ were in control group.

Basic clinical characteristics were compared among cases and controls illustrated in Table 1. No differences were found in age, BMI and waist circumference. Whereas statistically significant differences were found in fasting blood glucose and serum adiponectin levels. Figure: 1 illustrates the comparison of mean serum adiponectin levels of pre and postmenopausal groups among cases and control. Serum adiponectin was found significantly $(\mathrm{p}<0.05)$ higher in pre and postmenopausal healthy controls independently when compared to breast cancer cases.

Table: II represents the estimated risk (odds ratio) of breast cancer across the quartiles. Subjects were divided in to quartiles based on serum levels of adiponectin to find out the precise influence of serum adiponectin to
Table 1. Comparison of Breast Cancer Cases and Healthy Controls

\begin{tabular}{lccl}
\hline Clinical Characteristics & $\begin{array}{c}\text { Cases } \\
(\text { mean } \pm \text { SEM })\end{array}$ & $\begin{array}{c}\text { Control } \\
(\text { mean } \pm \text { SEM })\end{array}$ & p-value \\
\hline Age $(\mathrm{yr})$ & $46.15 \pm 0.80$ & $44.52 \pm 0.80$ & 0.153 \\
BMI $\left(\mathrm{Kg} / \mathrm{m}^{2}\right)$ & $21.61 \pm 0.31$ & $21.60 \pm 0.28$ & 0.97 \\
Waist Circumference $(\mathrm{cm})$ & $74.12 \pm 1.03$ & $75.45 \pm 0.83$ & 0.321 \\
Fasting Blood Glucose $(\mathrm{mg} / \mathrm{dl})$ & & \\
& $110.84 \pm 2.64 *$ & $101.25 \pm 2.64$ & 0.011 \\
Serum Adiponectin $(\mu \mathrm{g} / \mathrm{ml})$ & $6.63 \pm 0.11 *$ & $10.17 \pm 0.40$ & 0.000 \\
\hline
\end{tabular}

$* \mathrm{P}<0.05=$ statistically significant; $\mathrm{p}$-value was determined by student t-test

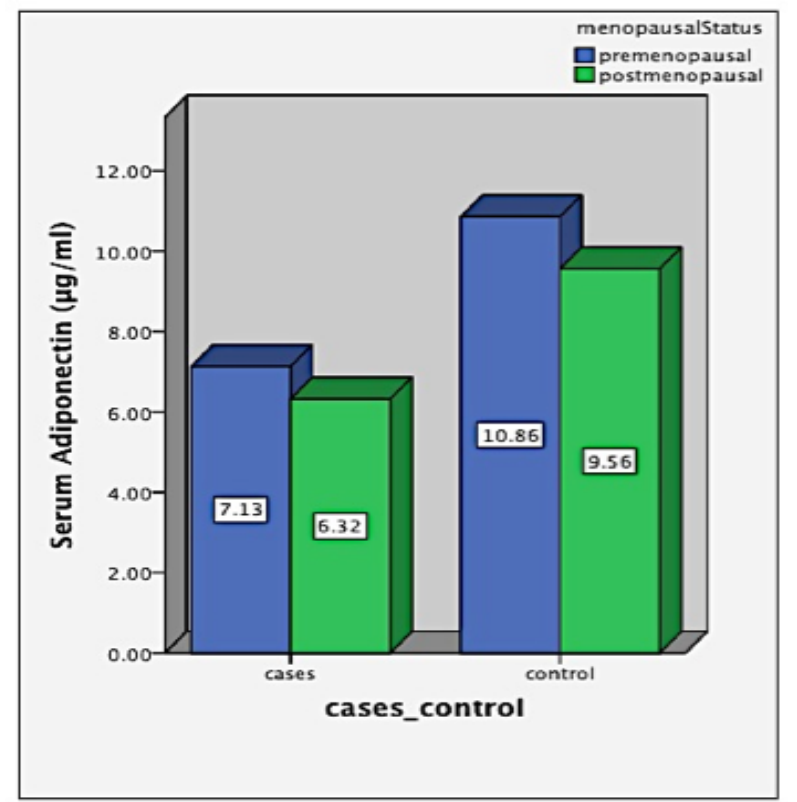

Figure 1. Comparison Of Serum Adiponectin Among Premenopausal And Postmenopausal Groups

Table 2. Risk Analysis for Breast Cancer by Quartiles of Serum Adiponectin

\begin{tabular}{lcrcll}
\hline \multicolumn{2}{l}{ Serum Adiponectin $(\mu \mathrm{g} / \mathrm{ml})$} & Cases & Control & OR & $95 \% \mathrm{Cl}$ \\
\cline { 1 - 3 } & Median (range) & $\mathrm{n}$ & $\mathrm{n}$ & & \\
\hline Q1 & $4.85(\leq 5.73)$ & 50 & 33 & $1^{\text {(vreference) }}$ & \\
Q2 & $6.36(5.74-6.88)$ & 67 & 25 & 1.76 & $(0.93-3.34)$ \\
Q3 & $8.05(6.89-9.50)$ & 50 & 37 & 0.89 & $(0.48-1.64)$ \\
Q4 & $13.87(>9.50)$ & 8 & 80 & $0.06 *$ & $(0.02-0.15)$
\end{tabular}

*p $\leq 0.05$; OR=Odds ratios; Risk of breast cancer was determined by odds ratio while significance of association between the exposure and disease was shown by $95 \%$ confidence interval (CI) for the odds ratio

the risk of breast. Lowest quartile $(<25$ th) was used as a reference for determining odds ratio and its difference within quartiles. It is observed that risk of breast cancer decreases with rising quartile. Q2 (25th-50th) has the highest $\mathrm{OR}=1.76$ (CI: 0.93- 3.34), Q3 (50th-75th) has $\mathrm{OR}=0.89$ (CI: 0.48-1.64), while subjects in the highest quartile Q4 (>75th) had significantly $(\mathrm{p}<0.05)$ decreased risk of breast cancer having $\mathrm{OR}=0.06$ (CI: 0.02-0.15), when compared to the lowest quartile.

\section{Discussion}

Protective role of elevated serum adiponectin levels from breast cancer is still under investigation with various 
propositions of molecular mechanisms involved. In this study, we have found significantly $(\mathrm{p}<0.05)$ higher levels of fasting blood glucose and lower serum adiponectin in breast cancer cases when compared with healthy controls. Serum adiponectin in breast cancer cases were also found lower in cases when subjects were distributed according to their menopausal status into premenopausal and postmenopausal.

Previous studies have reported inconsistent results. Study conducted among Malaysian women (Shahar et al., 2010) showed significantly higher fasting blood glucose and lower adiponectin levels in cases compared to controls, whereas another study conducted in Korea (Kang et al., 2007) demonstrated significantly higher fasting blood glucose in cases, but no significant difference in serum adiponectin when compared with healthy controls.

Demonstration of elevated fasting blood glucose association with increased risk of breast cancer by several studies (Stattin et al., 2007; Haseen et al., 2015) and finding of this study consensually signifies the fact that lower adiponectin level loses its effect of decreasing synthesis of glucose in liver and other tissues (Kadowaki and Yamauchi, 2005) leading to hyperglycemia. Therefore lower adiponectin levels led to the development of insulin resistance and compensatory chronic hyperinsulinemia, resulting in increased levels of bioavailable IGF1. This will promote cellular proliferation and inhibit apoptosis by up regulating the secretion of vascular endothelial growth factor, contributing thus to carcinogenesis (Calle and Kaaks, 2004).

This study has also further validated the fact that risk of breast cancer decreases as the levels of serum adiponectin rises. We have showed significantly lower risk $(\mathrm{OR}=0.06)$ of breast cancer as well as association $(95 \% \mathrm{CI}$ : $0.02-0.15$ ) between the elevated adiponectin serum level and lower risk of breast cancer in subjects having serum adiponectin greater than $9.50 \mu \mathrm{g} / \mathrm{ml}$. This finding may suggests that higher levels of serum adiponectin protects from carcinogenesis through masking of growth factors like IGF1, PDGF-BB, FGF, HB EGF and VEGF. Our findings are in accordance with other studies conducted in different parts of the world (Korner et al., 2007; Shahar et al., 2010; Karimi and Roshan, 2013) that may represents the validity of this finding without disparity among regions and ethnicity.

This study has limitations including those accompanying with the cross-sectional nature of the study design. Cases and controls were age, sex and BMI matched, nonetheless it was not adjusted for ethnicity, fasting blood glucose and other risk factors. Similar future study is advocated which should be population based, multi-centric and prospective to find out the causal relationship.

In conclusion, this case-control study has demonstrated statistically significant higher serum adiponectin levels in normal healthy controls when compared to breast cancer subjects. In addition, on distributing subjects in to quartiles, highest quartile was found to have lowest risk of breast cancer. These findings signify complex mechanism involving adipocyte-produced adiponectin cross talks and cell signaling in breast carcinogenesis, suggesting its therapeutic modulation as a possible preventive measure.

\section{Acknowledgements}

We would like to thank Department of Biochemistry, University of Karachi for funding the biochemical analysis of subjects in this study thru research grant for $\mathrm{PhD}$ students. The co-operation of patients and staff members of Breast Clinic, Surgical Unit-1, Civil Hospital Karachi Pakistan is also acknowledged.

\section{References}

Bhurgri Y (2004). Karachi cancer registry data--implications for the national cancer control program of pakistan. Asian Pac J Cancer Prev, 5, 77-82.

Calle EE, Kaaks R (2004) Overweight, obesity and cancer: epidemiological evidence and proposed mechanisms. Nat Rev Cancer, 4, 579-91.

Dean AG, Sullivan KM, Soe MM (2014). Openepi: open source epidemiologic statistics for public health, version. http:// www.OpenEpi.com, updated September 01,2014. Accessed September 04, 2014.

Ferlay J, Soerjomataram I, Dikshit R, et al (2015). Cancer incidence and mortality worldwide: sources, methods and major patterns in GLOBOCAN 2012. International Journal of Cancer, 136, 359-86.

Haseen SD, Khanam A, Sultan N, et al (2015). Elevated fasting blood glucose is associated with increased risk of breast cancer: outcome of case-control study conducted in karachi, Pakistan. Asian Pac J Cancer Prev, 16, 675-8.

IBM Corp. (2011). IBM SPSS Statistics for Windows, Version 20.0. Armonk, NY: IBM Corp.

Kadowaki T, Yamauchi T (2005). Adiponectin and adiponectin receptors. Endocr Rev, 26, 439-51.

Kadowaki T, Yamauchi T, Kubota N, et al (2006). Adiponectin and adiponectin receptors in insulin resistance, diabetes, and the metabolic syndrome. J Clin Invest, 116, 1784-92.

Kang JH, Yu BY, Youn DS (2007). Relationship of serum adiponectin and resistin levels with breast cancer risk. $J$ Korean Med Sci, 22, 117-21.

Karimi N, Roshan VD (2013). Change in adiponectin and oxidative stress after modifiable lifestyle interventions in breast cancer cases. Asian Pac J Cancer Prev, 14, 2845-50.

Kelesidis I, Kelesidis T, Mantzoros CS (2006). Adiponectin and cancer: a systematic review. Br J Cancer, 94, 1221-5.

Keramatinia A, Mousavi-Jarrahi SH, Hiteh M, Mosavi-Jarrahi A. (2014). Trends in incidence of breast cancer among women under 40 in Asia. Asian Pac J Cancer Prev, 15, 1387-90.

Korner A, Pazaitou-Panayiotou K, Kelesidis T, et al (2007). Total and high-molecular-weight adiponectin in breast cancer: in vitro and in vivo studies. J Clin Endocrinol Metab, 92, 1041-8.

Leclere B, Molinie F, Tretarre B, et al (2013). Trends in incidence of breast cancer among women under 40 in seven European countries: a GRELL cooperative study. Cancer Epidemiol, 37, 544-9.

Shahar S, Salleh RM, Ghazali AR, Koon PB, Mohamud, WN (2010). Roles of adiposity, lifetime physical activity and serum adiponectin in occurrence of breast cancer among Malaysian women in Klang Valley. Asian Pac J Cancer Prev, 11, 61-6.

Stattin P, Bjor O, Ferrari P (2007). Prospective study of hyperglycemia and cancer risk. Diabetes Care, 30, 561-7.

Torre LA, Bray F, Siegel RL, et al (2015). Global cancer statistics, 2012. CA: A Cancer J Clinicians, 65, 87-108. 
Syed Danish Haseen Ahmed et al

Wang Y, Lam KS, Xu JY, et al (2005) Adiponectin inhibits cell proliferation by interacting with several growth factors in an oligomerization-dependent manner. J Biol Chem, 280, 18341-7.

Yamauchi T, Kamon J, Ito Y, et al (2003). Cloning of adiponectin receptors that mediate antidiabetic metabolic effects. Nature, 423, 762-9. 